\title{
Entanglement of spatial regions vs. entanglement of particles
}

\author{
Swapnamay Mondal \\ Harish-Chandra Research Institute, \\ Chhatnag Road, Jhunsi, Allahabad 211019, India \\ E-mail: swapno@hri.res.in
}

ABSTRACT: Consider an arbitrary local quantum field theory with a gap or an arbitrary gapless free theory. We consider states in such a theory, that describe two entangled particles localized in disjoint regions of space. We show that in such a state, to leading order, Rényi entropies of spatial regions, containing only one of the particles are same as their quantum mechanical counterparts, after subtraction of vacuum contribution. Subleading corrections depend on overlap of wave functions. These results suggest that Von Neumann entropy of a spatial region, after subtraction of vacuum contribution, can serve as a measure of entanglement of indistinguishable particles in pure states.

Keywords: Field Theories in Higher Dimensions, Lattice Quantum Field Theory

ARXIV EPRINT: 1501.01743 


\section{Contents}

1 Introduction 1

2 Entanglement: spatial regions vs. particles 2

$\begin{array}{llr}3 & \text { Discussion } & 6\end{array}$

$\begin{array}{ll}\text { A Derivation of (2.10) } & 7\end{array}$

$\begin{array}{lr}\text { B Correction terms } & 8\end{array}$

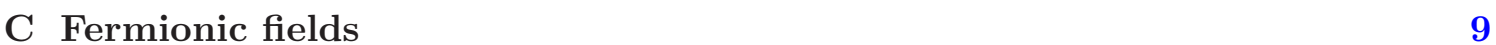

\section{Introduction}

What makes quantum mechanics mathematically nice is linearity. On the other hand what makes quantum mechanics physically somewhat counterintuitive is also linearity. The best manifestation of this is entanglement, non triviality of which has been noted as early as 1935 [1]. In last few decades it has also been realized that entanglement of quantum particles can be put to practical use. This is at the very heart of the subject of quantum information and communication. Recent years have witnessed widespread interest in another somewhat different aspect of entanglement, namely entanglement of spatial regions in quantum field theories. This has been found to be relevant in diverse areas of physics such as holography $[2,3]$, entropic c theorem $[4,5]$, quantum phase transitions [6-8] to name a few.

In this paper we ask a rather simple minded question, which is possibly of quite general interest. We consider a state in a general local quantum field theory that can be thought of as an entangled state of two localized particles. Then the entanglement (in the framework of quantum mechanics) of the emergent particles, is clearly a relevant notion in this context. On the other hand in the framework of field theory, the natural notion is entanglement of spatial regions (containing one of the localized particles). Hence one would expect some relation between these two notions. To our knowledge such a relation has not been investigate previously. ${ }^{1}$ We address this question in this paper and show that such a relation exists for any local quantum field theory. Since field theory is a more fundamental

\footnotetext{
${ }^{1}$ Rényi and Von Neumann entropies of locally excited states have been studied in $[9,10]$ for conformal field theories. But there one is mainly concerned about time evolution of entanglement. In [11] mutual Rényi information of locally excited entangled states was considered and it was noted that this admits a quantum mechanical interpretation. We show this for Rényi entropies themselves and extract somewhat different physics than these papers.
} 
description of elementary particles than quantum mechanics, this also provides a finer notion of entanglement of particles. ${ }^{2}$

Our results may have interesting bearing in black hole information paradox [12, 13], more precisely on Mathur's argument [14] and AMPS argument [15]. This is because these arguments are based on quantum mechanical notion of entanglement, whereas the natural framework for the problem is quantum field theory in curved space times. To leading order, it would not matter, nevertheless it may be interesting to explore small corrections due to this.

Our paper is organized as follows: in section 2, we derive our main result (2.15), which is relating Rényi entropies of spatial regions to those of quantum particles. In 3 we discuss possible usage of our results for the problem of entanglement of indistinguishable particles.

\section{Entanglement: spatial regions vs. particles}

Consider a state of the following form

$$
|\Psi\rangle=N\left(a O_{i} \tilde{O}_{j}+b O_{k} \tilde{O}_{l}\right)|0\rangle .
$$

$N$ is a normalization constant and $O_{i}$ denotes creation operator for a mode corresponding to the spatial wave function $\phi_{1}(x)$ (which is concentrated around the point $x_{1}$ ) and internal index $i, \tilde{O}_{j}$ denotes creation operator for a mode corresponding to the spatial wave function $\phi_{2}(x)$ (which is concentrated around the point $x_{2}$ ) and internal index $j$. Similar statements hold for $O_{k}, \tilde{O}_{l}$.

For a wave function $\phi(x)$, or equivalently $\tilde{\phi}(k)$, the corresponding state is $\int \frac{d^{D} k}{\sqrt{2 E_{k}}} \tilde{\phi}(k)|k\rangle$, where $|k\rangle$ is the one particle state of momentum $k$. For free theories (both gapped and gapless) we have explicit construction of such states in terms of creation operators. For gapped interacting field theories, we still have unambiguous notion of one particle momentum eigenstates $|k\rangle$. In this case we can define "creation operator" $a_{k}^{\dagger}$ by $|k\rangle=: a_{k}^{\dagger}|0\rangle$, with $|0\rangle$ being the interacting vacuum. Since one particle states are orthogonal to the bound states as well as continnum of multi-particle states, we have $a_{k}|\alpha\rangle=0$ for any bound/ multi-particle state $|\alpha\rangle$. Thus $O, \tilde{O}$ are simply suitable linear superpositions of these $a_{k}^{\dagger}$-s. However the notion of one particle states is a bit ambiguous for gapless interacting theories. This is becasue being interacting one does not have an explicit creation operator to start with and being gapless it is not clear how to extract one particle states from continuous spectrum. Hence we would exclude such theories form our analysis.

We choose the following normalization ${ }^{3}$ for $O-\mathrm{s}$

$$
\left\langle 0\left|O_{i}^{\dagger} O_{j}\right| 0\right\rangle=\delta_{i j}=\left\langle 0\left|\tilde{O}_{i}^{\dagger} \tilde{O}_{j}\right| 0\right\rangle .
$$

Now if the wave functions $\phi_{1}(x)$ and $\phi_{2}(x)$ are entirely supported in disjoint regions $A_{1}$ and $A_{2}$, such a state describes an entangled state of two particles, distinguishable by their

\footnotetext{
${ }^{2}$ In spirit, this is similar to various physical quantities, e.g. magnetic moment of electron, which have a coarser description in quantum mechanics and a finer one in field theory. Although for entanglement the finery is an artefact of the state under consideration, rather than the theory.

${ }^{3}$ Note as $O$-s contain only creation operators, they (anti)commute among themselves.
} 
positions. This may then be described, within the framework of quantum mechanics of distinguishable particles, as

$$
\left|\psi_{\text {spin }}\right\rangle=\frac{1}{\sqrt{|a|^{2}+|b|^{2}}}(a|i\rangle|j\rangle+b|k\rangle|l\rangle),
$$

with the spatial wave functions $\phi_{1}(x)$ and $\phi_{2}(x)$ serving as particle labels.

A natural question to ask is that what is the connection between entanglement of (2.3) and entanglement of the region say $A_{1}$, in the state (2.1)? We answer this question in this paper for arbitrary local quantum field theory with a gap and for arbitrary local gapless free theory.

Bipartite entanglement of a pure state is quantified by the Von Nuemann entropy $S(\rho)=-\operatorname{Tr} \rho \log \rho$, where $\rho$ is the density matrix of any of the parties. However for our purpose, Rényi entropies prove more useful. $n^{\text {th }}$ Rényi entropy of a density matrix $\rho$ is given by

$$
S_{n}(\rho)=\frac{1}{1-n} \log \operatorname{Tr} \rho^{n},
$$

where $\rho$ is the density matrix of any of the parties. A party would stand for a paricle (or a collection of particles) while discussing a quantum mechanical state and a region of space while discussing a field theory state.

For computational reasons it is even more advantageous to look at the following quantity

$$
R_{n}(\rho):=e^{(1-n) S_{n}(\rho)}=\operatorname{Tr} \rho^{n} .
$$

First we note that we can express $R_{n}$ as

$$
R_{n}(\rho)=\operatorname{Tr}_{n}\left[\rho^{\otimes n} E^{(n)}\right]
$$

with

$$
E_{j_{1} \ldots j_{n} ; i_{1} \ldots i_{n}}^{(n)}=\prod_{k=1}^{n} \delta_{j_{k}, i_{k+1}} .
$$

$\operatorname{Tr}_{n}$ stands for trace over $\mathcal{H}_{1}^{\otimes n}$, where $\mathcal{H}_{1}$ is the Hilbert space of the subsystem under consideration. The indices $\left\{i_{k}, j_{k}\right\}$ take discreet values if we are discussing a quantum mechanical state and continuous values if we are discussing a field theoretic state. Note that (2.6) does not fix $E^{(n)}$ uniquely.

As defined above, $E^{(n)}$ is a linear operator on $\mathcal{H}_{1}^{\otimes n}$. One can easily extend its action on the full tensor product Hilbert space $\left(\mathcal{H}_{1} \otimes \mathcal{H}_{2}\right)^{\otimes n}$ by defining

$$
E_{j_{1}, b_{1} \ldots j_{n}, b_{n} ; i_{1}, a_{1} \ldots i_{n}, a_{n}}^{(n)}=\prod_{k=1}^{n} \delta_{j_{k}, i_{k+1}} \delta_{a_{k}, b_{k}},
$$

where $i_{k}, j_{k}$ are the indices of $k$-th copy of $\mathcal{H}_{1}$ and $a_{k}, b_{k}$ are the indices of $k$-th copy of $\mathcal{H}_{2}$. In fact if $\rho_{1}=\operatorname{Tr}_{2} \rho_{12}$, then

$$
R_{n}\left(\rho_{1}\right)=\operatorname{Tr}_{n}\left[\rho_{12}^{\otimes n} E^{(n)}\right],
$$

where now trace is over $\left(\mathcal{H}_{1} \otimes \mathcal{H}_{2}\right)^{\otimes n}$. From now on we will suppress the subscripts on trace and assume it is clear from the context. 
For us $\rho_{12}=|\Psi\rangle\langle\Psi|$ (with $|\Psi\rangle$ given by (2.1)) and subsystem that we are interested in, is a spatial region $\Omega$. Thus $\rho_{1}=\rho_{\Omega}=\operatorname{Tr}_{\Omega_{c}}|\Psi\rangle\langle\Psi|, \Omega_{c}$ being the complementary region of $\Omega$. The operator $E_{\Omega}^{(n)}$ has been studied in great details in a recent paper [11] and we will heavily borrow their results. If we denote local field variable at point $a$ as $q_{a}$, then $E_{\Omega}^{(n)}$ reads

$E_{\Omega}^{(n)}=\int \prod_{j=1}^{n} \prod_{a \in \Omega} d q_{a}^{(j)} \prod_{b \in \Omega} d q_{b}^{\left(j^{\prime}\right)}\left|\left\{q_{b}^{1^{\prime}} \ldots q_{b}^{n^{\prime}}\right\}\right\rangle\left\langle\left\{q_{a}^{1} \ldots q_{a}^{n}\right\}\right| \delta\left(q_{b}^{\left(1^{\prime}\right)}-q_{a}^{(2)}\right) \ldots \delta\left(q_{b}^{\left(n^{\prime}\right)}-q_{a}^{(1)}\right) \times I_{\Omega_{c}}^{n}$,

where $I_{\Omega_{c}}^{n}$ is identity operator on $\mathcal{H}_{\Omega_{c}}^{\otimes n}, \mathcal{H}_{\Omega_{c}}$ being the Hilbert space associated with field variables living in the region $\Omega_{c}$. Perhaps a more comprehensible way to express $E_{\Omega}^{(n)}$ is the following

$$
\begin{aligned}
E_{\Omega}^{(n)}= & \int D \phi_{\Omega}^{1} \ldots D \phi_{\Omega}^{n} D \phi_{\Omega}^{1^{\prime}} \ldots D \phi_{\Omega}^{n^{\prime}} D \phi_{\Omega_{c}}^{1} \ldots D \phi_{\Omega_{c}}^{n} \delta\left(\phi_{\Omega}^{1^{\prime}}-\phi_{\Omega}^{2}\right) \ldots \delta\left(\phi_{\Omega}^{n^{\prime}}-\phi_{\Omega}^{1}\right) \\
& \left|\phi_{\Omega}^{1^{\prime}} \ldots \phi_{\Omega}^{n^{\prime}}\right\rangle\left\langle\phi_{\Omega}^{1} \ldots \phi_{\Omega}^{n}|\otimes| \phi_{\Omega_{c}}^{1} \ldots \phi_{\Omega_{c}}^{n}\right\rangle\left\langle\phi_{\Omega_{c}}^{1} \ldots \phi_{\Omega_{c}}^{n}\right| \\
& =\int D \phi_{\Omega}^{1} \ldots D \phi_{\Omega}^{n} D \phi_{\Omega_{c}}^{1} \ldots D \phi_{\Omega_{c}}^{n}\left|\phi_{\Omega}^{2} \ldots \phi_{\Omega}^{1}\right\rangle\left\langle\phi_{\Omega}^{1} \ldots \phi_{\Omega}^{n}|\otimes| \phi_{\Omega_{c}}^{1} \ldots \phi_{\Omega_{c}}^{n}\right\rangle\left\langle\phi_{\Omega_{c}}^{1} \ldots \phi_{\Omega_{c}}^{n}\right| .
\end{aligned}
$$

Here $\left|\phi_{\Omega}\right\rangle$ denotes the state corresponding to a smooth field configuration over region $\Omega$ and similar statement holds for $\left|\phi_{\Omega_{c}}\right\rangle$. These are analogs of position eigenstates in quantum mechanics. $E_{\Omega}^{(n)}$ has many nice properties, as explored in [11]. The one particularly useful for us is

$$
\left\langle\psi_{1} \ldots \psi_{n}\left|E_{\Omega}^{(n)}\right| \chi_{1} \ldots \chi_{n}\right\rangle=\left\langle\chi_{2} \ldots \chi_{1}\left|E_{\Omega_{c}}^{(n)}\right| \psi_{1} \ldots \psi_{n}\right\rangle^{*} .
$$

A derivation of this property is given in A.

Armed with these, we set out to compute $R_{n}\left(\rho_{\Omega}\right)$. We consider a bosonic theory to start with. The main result of this section, namely (2.15) would hold both for bosonic and fermionic fields. The corrections to this (disussed in B) would differ though. First we note

$$
R_{n}\left(\rho_{\Omega}\right)=\operatorname{Tr}\left(\left|\Psi^{\otimes n}\right\rangle\left\langle\Psi^{\otimes n}\right| E_{\Omega}^{(n)}\right)=\left\langle\Psi^{\otimes n}\left|E_{\Omega}^{(n)}\right| \Psi^{\otimes n}\right\rangle .
$$

This contains $4^{n}$ terms, each of the following form

$$
\begin{aligned}
& \left\langle 0\left|\left(\tilde{O}_{j_{n}}^{(n)}\right)^{\dagger}\left(O_{i_{n}}^{(n)}\right)^{\dagger} \ldots\left(\tilde{O}_{j_{n}}^{(n)}\right)^{\dagger}\left(O_{i_{n}}^{(n)}\right)^{\dagger} E_{\Omega}^{(n)} O_{p_{1}}^{(1)} \tilde{O}_{q_{1}}^{(1)} \ldots O_{p_{1}}^{(1)} \tilde{O}_{q_{1}}^{(1)}\right| 0\right\rangle \\
& =\left\langle 0\left|\left\{\left(\tilde{O}_{j_{n}}^{(n)}\right)^{\dagger} \ldots\left(\tilde{O}_{j_{1}}^{(1)}\right)^{\dagger}\right\}\left\{\left(O_{i_{n}}^{(n)}\right)^{\dagger} \ldots\left(O_{i_{1}}^{(1)}\right)^{\dagger}\right\} E_{\Omega}^{(n)}\left\{O_{p_{1}}^{(1)} \ldots O_{p_{n}}^{(n)}\right\}\left\{\tilde{O}_{q_{1}}^{(1)} \ldots \tilde{O}_{q_{n}}^{(n)}\right\}\right| 0\right\rangle .
\end{aligned}
$$

Here we have ignored the normalization factor $N^{2 n}$. To shorten the expressions, we introduce some notations

$$
\begin{aligned}
\left(O_{i_{n}}^{(n)}\right)^{\dagger} \ldots\left(O_{i_{1}}^{(1)}\right)^{\dagger} & =: O_{i}^{\dagger} \\
\left(\tilde{O}_{j_{n}}^{(n)}\right)^{\dagger} \ldots\left(\tilde{O}_{j_{1}}^{(1)}\right)^{\dagger} & =: \tilde{O}_{j}^{\dagger} \\
O_{p_{1}}^{(1)} \ldots O_{p_{n}}^{(n)} & =: O_{p} \\
\tilde{O}_{q_{1}}^{(1)} \ldots \tilde{O}_{q_{n}}^{(n)} & =: O_{q} .
\end{aligned}
$$


So we have

$$
\left\langle 0\left|\tilde{O}_{j}^{\dagger} O_{i}^{\dagger} E_{\Omega}^{(n)} O_{p} \tilde{O}_{q}\right| 0\right\rangle .
$$

Remember, in present notation all the $O, O^{\dagger}$-s are clusters of $n$ creation/annihilation operators. After some more steps, which we describe in appendix B, to set the clutter aside, this can be written as

$$
\left\langle 0\left|E_{\Omega}^{(n)}\right| 0\right\rangle \times \delta_{p_{2}, i_{1}} \ldots \delta_{p_{1}, i_{n}} \times \delta_{j_{1} q_{1}} \ldots \delta_{j_{n} q_{n}}+\text { other pieces } .
$$

"Other pieces" are discussed in detail in appendix B. For now we just mention the relevant property of these pieces. They are small when the region $\Omega$ is chosen such that $\phi_{1}(x)$ is mostly supported inside $\Omega$ and $\phi_{2}(x)$ is supported mostly outside $\Omega$. Thus we concentrate on the leading piece. When this piece is added for all the $4^{n}$ terms, one ends up with

$$
N^{2 n}\left\langle 0\left|E_{\Omega}^{(n)}\right| 0\right\rangle\left(|a|^{2 n}+|b|^{2 n}\right)=\left\langle 0\left|E_{\Omega}^{(n)}\right| 0\right\rangle\left(N \sqrt{\left|a^{2}\right|+|b|^{2}}\right)^{2 n} R_{n}\left(\rho_{1}^{Q M}\right),
$$

where $\rho_{1}^{Q M}$ is the density matrix of the first particle in the state (2.3). If $O$ and $\tilde{O}$ are supported in disjoint regions, which is the case considered, $N \sim\left(\sqrt{|a|^{2}+|b|^{2}}\right)^{-1}$. Thus

$$
\begin{aligned}
R_{n}\left(\rho_{\Omega}(\Psi)\right) & \sim R_{n}\left(\rho_{\Omega}(0)\right) \times R_{n}\left(\rho_{1}^{Q M}(\psi)\right) \\
\text { or, } S_{n}\left(\rho_{\Omega}(\Psi)\right)-S_{n}\left(\rho_{\Omega}(0)\right) & \sim S_{n}\left(\rho_{1}^{Q M}(\psi)\right),
\end{aligned}
$$

to leading order. "Other pieces" mentioned in (2.13) and deviation of $N$ from $\left(\left|a^{2}\right|+\right.$ $\left.|b|^{2}\right)^{-1 / 2}$ constitute corrections to this. We mention one interestig property of one of the correction terms (see B for detail). Among "other pieces", second piece of (B.1) contains a factor of $\prod_{k} j_{k} p_{k}$. This will give non-zero contribution if $i=l$ or $j=k$ or $i=j$ or $k=l$ in $(2.1)$.

A little thought would convince the reader that if we consider a more general state than (2.1), i.e. a state of the form

$$
|\Psi\rangle=N \sum_{\alpha} a_{\alpha} O_{i_{\alpha}} \tilde{O}_{j_{\alpha}}|0\rangle
$$

(2.15) would still hold. In fact our results continue to hold when instead of a single particle each subsystem contains a bunch of particles localized in some region. Now $O, \tilde{O}$ would contain a bunch of creation operators, but that does not change any of the steps.

Another interesting case is of occupation number entanglement. In this case, one would consider states of the form

$$
|\Psi\rangle=\sum_{n} a_{n} O^{n} \tilde{O}^{n}|0\rangle .
$$

In occupation number eigenbasis (and assuming orthogonality of the modes occupied) the above state would read

$$
|\psi\rangle=\sum_{n} a_{n}|n, n\rangle .
$$


We can repeat the very same steps as in B. Corresponding to each Krönecker delta in spin indices in B, now we would have a Krönecker delta in occupation number. Hence for present purpose they play the role of spin indices and (2.15) alongwith correction terms also holds for occupation number entanglement.

\section{Discussion}

These results prove useful for a problem in quantum mechanics, namely the problem of entanglement of indistinguishable particles. ${ }^{4}$ If wave functions of various particles are nearly orthogonal they can be assigned different values of some observable and the particles can effectively be labelled by the value of that observable. Afterwards one can discuss entanglement of those particles treating them as distinguishable particles. E.g. electrons stuck to various lattice sites can be labelled by the site it sits on (i.e. position) and they can be entangled through spin.

When wave functions start overlaping such labelling becomes ambiguous and the inherently indistinguishable nature of fundamental particles become important. The primary difficulty in discussing entanglement in such situation is identifying distinguishable subsystems, for particles are no more distinguishable. It is useful to take a field theoretic view of the situation, for then spatial regions serve as natural subsystems. When wavefunctions are localized around different positions, one can choose a region $\Omega$ containing only a single particle. The subsystem $\Omega$ then corresponds to that particle. This can be made quantitative if one finds a field theoretic quantity $\tilde{S}$, which is nearly equal to the Von Neumann entropy of the quantum mechanical density matrix of that particle. Even if the wave functions overlap, $\tilde{S}$ remains well defined. Thus provided $\tilde{S}$ is finite, it would be a natural candidate for entanglement of indistinguishable particles. The subsystem $\Omega$ can not clearly be associated with any particle though.

(2.15) suggests $S\left(\rho_{\Omega}(\Psi)\right)-S\left(\rho_{\Omega}(0)\right)$ is the natural candidate for $\tilde{S}$, where we have used notations of last section and $S(\rho)$ denotes the Von Neumann entropy of the density matrix $\rho$. This quantity has the correct limit as the wavefunction overlap approaches zero. Given that Von Neumann entropy quantifies entanglement in pure states [24], $S\left(\rho_{\Omega}(\Psi)\right)$ indeed quantifies entanglement. Further subtraction of vacuum contribution renders it finite.

(2.15) also tells that $S\left(\rho_{\Omega}(\Psi)\right)-S\left(\rho_{\Omega}(0)\right)$ would in general differ from the naive quantum mechanical answer $S$, the difference being given by the "correction terms" described in B (for Rényi entropies). It should be noted that these corrections are never exactly zero, since there are no localized particles in a local quantum field theory [25]. We interpret these corrections as field theoretic corrections to naive quantum mechanical notion of entanglement. Due to these corrections, one would in general require an infinite dimensional density matrix to account for the vaccum subtracted Von Neumann and Rényi entropies.

\footnotetext{
${ }^{4}$ Many attempts have been made in recent years to generalize the notion of entanglement for indistinguishable particles $[16,17],[18-21]$. Look at [22, 23] for recent review and references there in. These approaches mostly explore two directions, namely tensor product structure of the Hilbert space and occupation number entanglement between various modes (which is indeed well suited for many systems considered in laboratory).
} 
In the spectrum of this density matrix field theoretic corrections correspond to infnitely many small eigenvalues. In terms of the entanglement spectrum, ${ }^{5}$ this means field theoretic effects are encoded in "high energy states", i.e. quantum mechanics serves as a "low energy effective theory" in the context of entanglement.

These correction terms also lead to interesting properties for the lattice analog of $\tilde{S}$, namely site entanglement $[16,17]$. E.g. a single electron with wavefunction supported at more than one lattice site looks like an entangled state! ${ }^{6}$ However bizarre, such entanglement has actually been used in teleportation [30] and therefore physical. Given this it is natural to wonder whether the continuum analog of this can have some practical use as well. E.g. due to the field theoretic corrections, entanglement of a "single" electron can exceed $\log 2$. It would be interesting to explore possible implications of this for quantum information and communication. ${ }^{7}$

\section{Acknowledgments}

I am indebted to my friend Pinaki Banerjee for collaboration in earlier part of this work and many subsequent discussions. My special thanks to Ashoke Sen for various illuminating discussions and valuable comments on the manuscript. I would also like to thank Nilay Kundu, Arunabha Saha and whole QIC group at HRI, especially H.S. Dhar, M.N. Bera, Aditi Sen(De) and Avijit Misra for useful conversations and comments. Lastly I thank people of India for their generous support for research in theoretical physics. This work has been supported by DAE project 12-R\&D-HRI-5.02-0303.

\section{A Derivation of (2.10)}

To make the logic more tractable we derive (2.10) for $n=3$.

$$
\begin{aligned}
E_{\Omega}^{(3)}= & \int D \phi_{\Omega}^{1^{\prime}} D \phi_{\Omega}^{2^{\prime}} D \phi_{\Omega}^{3^{\prime}} D \phi_{\Omega}^{1} D \phi_{\Omega}^{2} D \phi_{\Omega}^{3} \delta\left(\phi_{\Omega}^{1^{\prime}}-\phi_{\Omega}^{2}\right) \delta\left(\phi_{\Omega}^{2^{\prime}}-\phi_{\Omega}^{3}\right) \delta\left(\phi_{\Omega}^{3^{\prime}}-\phi_{\Omega}^{1}\right)\left|\phi_{\Omega}^{1^{\prime}} \phi_{\Omega}^{2^{\prime}} \phi_{\Omega}^{3^{\prime}}\right\rangle\left\langle\phi_{\Omega}^{1} \phi_{\Omega}^{2} \phi_{\Omega}^{3}\right| \\
& \times \int D \phi_{\Omega_{c}}^{1} D \phi_{\Omega_{c}}^{2} D \phi_{\Omega_{c}}^{3}\left|\phi_{\Omega_{c}}^{1} \phi_{\Omega_{c}}^{2} \phi_{\Omega_{c}}^{3}\right\rangle\left\langle\phi_{\Omega_{c}}^{1} \phi_{\Omega_{c}}^{2} \phi_{\Omega_{c}}^{3}\right| \\
= & \int D \phi_{\Omega}^{1} D \phi_{\Omega}^{2} D \phi_{\Omega}^{3} D \phi_{\Omega_{c}}^{1} D \phi_{\Omega_{c}}^{2} D \phi_{\Omega_{c}}^{3}\left|\phi_{\Omega}^{2} \phi_{\Omega}^{3} \phi_{\Omega}^{1}\right\rangle\left\langle\phi_{\Omega}^{1} \phi_{\Omega}^{2} \phi_{\Omega}^{3}|\times| \phi_{\Omega_{c}}^{1} \phi_{\Omega_{c}}^{2} \phi_{\Omega_{c}}^{3}\right\rangle\left\langle\phi_{\Omega_{c}}^{1} \phi_{\Omega_{c}}^{2} \phi_{\Omega_{c}}^{3}\right| .
\end{aligned}
$$

Now we can express any state $|\psi\rangle$ as

$$
|\psi\rangle=\int D \phi_{\Omega} D \phi_{\Omega_{c}} \psi\left[\phi_{\Omega}, \phi_{\Omega_{c}}\right]\left|\phi_{\Omega}\right\rangle \otimes\left|\phi_{\Omega_{c}}\right\rangle
$$

\footnotetext{
${ }^{5}$ Modular Hamiltonian $H$ for a given density matrix $\rho$ is defined as $\rho=e^{-H}$. Previously the spectrum of $H$, called "entaglement spectrum", has been argued to carry footprints of topological order [26], in the context of fractional quantum Hall effect. The low energy states of the entanglement spectrum become gapless, as the system becomes topologically ordered.

${ }^{6}$ Another strange feature is that the entanglement (through spin) of two electrons at same site (distinguished by orbitals quantum number) is invisible to site entanglement. These points have previously been noted in [16, 27]. Also look at [28, 29].

${ }^{7}$ We thank Arunabha Saha for pointing out this possibility.
} 
Hence

$$
\begin{aligned}
\left\langle\psi_{1} \psi_{2} \psi_{3}\left|E_{\Omega}^{(3)}\right| \chi_{1} \chi_{2} \chi_{3}\right\rangle & \\
= & \int D \phi_{\Omega}^{1} D \phi_{\Omega}^{2} D \phi_{\Omega}^{3} D \phi_{\Omega_{c}}^{1} D \phi_{\Omega_{c}}^{2} D \phi_{\Omega_{c}}^{3} \\
& \psi_{1}^{*}\left[\phi_{\Omega}^{2}, \phi_{\Omega_{c}}^{1}\right] \psi_{2}^{*}\left[\phi_{\Omega}^{3}, \phi_{\Omega_{c}}^{2}\right] \psi_{3}^{*}\left[\phi_{\Omega}^{1}, \phi_{\Omega_{c}}^{3}\right] \chi_{1}\left[\phi_{\Omega}^{1}, \phi_{\Omega_{c}}^{1}\right] \chi_{2}\left[\phi_{\Omega}^{2}, \phi_{\Omega_{c}}^{2}\right] \chi_{3}\left[\phi_{\Omega}^{3}, \phi_{\Omega_{c}}^{3}\right] \\
= & {\left[\int D \phi_{\Omega}^{1} D \phi_{\Omega}^{2} D \phi_{\Omega}^{3} D \phi_{\Omega_{c}}^{1} D \phi_{\Omega_{c}}^{2} D \phi_{\Omega_{c}}^{3}\right.} \\
& \left.\chi_{1}^{*}\left[\phi_{\Omega}^{1}, \phi_{\Omega_{c}}^{1}\right] \chi_{2}^{*}\left[\phi_{\Omega}^{2}, \phi_{\Omega_{c}}^{2}\right] \chi_{3}^{*}\left[\phi_{\Omega}^{3}, \phi_{\Omega_{c}}^{3}\right] \psi_{1}\left[\phi_{\Omega}^{2}, \phi_{\Omega_{c}}^{1}\right] \psi_{2}\left[\phi_{\Omega}^{3}, \phi_{\Omega_{c}}^{2}\right] \psi_{3}\left[\phi_{\Omega}^{1}, \phi_{\Omega_{c}}^{3}\right]\right]^{*} \\
= & {\left[\int D \phi_{\Omega_{c}}^{1} D \phi_{\Omega_{c}}^{2} D \phi_{\Omega_{c}}^{3} D \phi_{\Omega}^{1} D \phi_{\Omega}^{2} D \phi_{\Omega}^{3}\right.} \\
& \left.\chi_{2}^{*}\left[\phi_{\Omega_{c}}^{2}, \phi_{\Omega}^{1}\right] \chi_{3}^{*}\left[\phi_{\Omega_{c}}^{3}, \phi_{\Omega}^{2}\right] \chi_{1}^{*}\left[\phi_{\Omega_{c}}^{1}, \phi_{\Omega}^{3}\right] \psi_{1}\left[\phi_{\Omega_{c}}^{1}, \phi_{\Omega}^{1}\right] \psi_{2}\left[\phi_{\Omega_{c}}^{2}, \phi_{\Omega}^{2}\right] \psi_{3}\left[\phi_{\Omega_{c}}^{3}, \phi_{\Omega}^{3}\right]\right]^{*}
\end{aligned}
$$

(redefining $\phi_{\Omega}^{i}$ as $\phi_{\Omega_{c}}^{i-1}$ and thinking of $\phi_{\Omega_{c}}^{i}$ as the region of interest.)

$=\left\langle\chi_{2} \chi_{3} \chi_{1}\left|E_{\Omega_{c}}^{(3)}\right| \psi_{1} \psi_{2} \psi_{3}\right\rangle^{*}$.

In writing $\psi\left[\phi_{\Omega}, \phi_{\Omega_{c}}\right]$ as $\psi\left[\phi_{\Omega_{c}}, \phi_{\Omega}\right]$ we are just thinking $\mathcal{H}_{\Omega} \otimes \mathcal{H}_{\Omega_{c}}$ as $\mathcal{H}_{\Omega_{c}} \otimes \mathcal{H}_{\Omega}$, meaning now $\Omega_{c}$ is our region of interest rather than $\Omega$.

Similar derivation follows for general $n$ and we have

$$
\left\langle\psi_{1} \ldots \psi_{n}\left|E_{\Omega}^{(n)}\right| \chi_{1} \ldots \chi_{n}\right\rangle=\left\langle\chi_{2} \ldots \chi_{1}\left|E_{\Omega_{c}}^{(n)}\right| \psi_{1} \ldots \psi_{n}\right\rangle^{*}
$$

\section{B Correction terms}

Now we discuss the leftover "other pieces" in (2.13).

$$
\left\langle 0\left|\tilde{O}_{j}^{\dagger} O_{i}^{\dagger} E_{\Omega}^{(n)} O_{p} \tilde{O}_{q}\right| 0\right\rangle=\left\langle 0\left|O_{i}^{\dagger} E_{\Omega}^{(n)} O_{p} \tilde{O}_{j}^{\dagger} \tilde{O}_{q}\right| 0\right\rangle+\left\langle 0\left|\left[\tilde{O}_{j}^{\dagger}, O_{i}^{\dagger} E_{\Omega}^{(n)} O_{p}\right] \tilde{O}_{q}\right| 0\right\rangle
$$

$1^{\text {st }}$ piece in (B.1) can be further simplified as

$$
\left\langle 0\left|O_{i}^{\dagger} E_{\Omega}^{(n)} O_{p} \tilde{O}_{j}^{\dagger} \tilde{O}_{q}\right| 0\right\rangle=\left\langle 0\left|O_{i}^{\dagger} E_{\Omega}^{(n)} O_{p}\right| \alpha\right\rangle\left\langle\alpha\left|\tilde{O}_{j}^{\dagger} \tilde{O}_{q}\right| 0\right\rangle,
$$

where we have inserted a complete set $^{8}$ of states $|\alpha\rangle\langle\alpha|$. Only the $|\alpha\rangle=|0\rangle$ term survives, since for all others terms we have $\left\langle\alpha\left|\tilde{O}_{j}^{\dagger} \tilde{O}_{q}\right| 0\right\rangle=0$.

\footnotetext{
${ }^{8}$ For interacting gapless theories there could be confusion regarding which of $\{|\alpha\rangle\}$ represent one particle states. But we are excluding such theories from our analysis.
} 
We concentrate on the piece $\left\langle 0\left|O_{i}^{\dagger} E_{\Omega}^{(n)} O_{p}\right| 0\right\rangle$.

$$
\begin{aligned}
\langle 0| & O_{i}^{\dagger} E_{\Omega}^{(n)} O_{p}|0\rangle \\
= & \left\langle 0\left|\left\{\left(O_{i_{n}}^{(n)}\right)^{\dagger} \ldots\left(O_{i_{1}}^{(1)}\right)^{\dagger}\right\} E_{\Omega}^{(n)}\left\{O_{p_{1}}^{(1)} \ldots O_{p_{n}}^{(n)}\right\}\right| 0\right\rangle \\
= & \left\langle\psi_{i_{1}}, \ldots, \psi_{i_{n}}\left|E_{\Omega}^{(n)}\right| \psi_{p_{1}}, \ldots, \psi_{p_{n}}\right\rangle \\
= & \left\langle\psi_{p_{2}}, \ldots, \psi_{p_{1}}\left|E_{\Omega_{c}}^{(n)}\right| \psi_{i_{1}}, \ldots, \psi_{i_{n}}\right\rangle^{*} \\
= & \left\langle 0\left|\left\{\left(O_{p_{1}}^{(n)}\right)^{\dagger} \ldots\left(O_{p_{2}}^{(1)}\right)^{\dagger}\right\} E_{\Omega_{c}}^{(n)}\left\{O_{i_{1}}^{(1)} \ldots O_{i_{n}}^{(n)}\right\}\right| 0\right\rangle^{*} \\
= & \left\langle 0\left|\left\{\left(O_{p_{1}}^{(n)}\right)^{\dagger} \ldots\left(O_{p_{2}}^{(1)}\right)^{\dagger}\right\}\left\{O_{i_{1}}^{(1)} \ldots O_{i_{n}}^{(n)}\right\}\right| n\right\rangle^{*}\left\langle n\left|E_{\Omega_{c}}^{(n)}\right| 0\right\rangle^{*} \\
& +\left\langle 0\left|\left\{\left(O_{p_{1}}^{(n)}\right)^{\dagger} \ldots\left(O_{p_{2}}^{(1)}\right)^{\dagger}\right\}\left[E_{\Omega_{c}}^{(n)},\left\{O_{i_{1}}^{(1)} \ldots O_{i_{n}}^{(n)}\right\}\right]\right| 0\right\rangle^{*} \\
= & \left\langle 0\left|E_{\Omega}^{(n)}\right| 0\right\rangle \delta_{p_{2}, i_{1}} \ldots \delta_{p_{1}, i_{n}}+\left\langle 0\left|\left\{\left(O_{p_{1}}^{(n)}\right)^{\dagger} \ldots\left(O_{p_{2}}^{(1)}\right)^{\dagger}\right\}\left[E_{\Omega_{c}}^{(n)},\left\{O_{i_{1}}^{(1)} \ldots O_{i_{n}}^{(n)}\right\}\right]\right| 0\right\rangle^{*} .
\end{aligned}
$$

Gathering all these, we see

$$
\begin{aligned}
\left\langle 0\left|\tilde{O}_{j}^{\dagger} O_{i}^{\dagger} E_{\Omega}^{(n)} O_{p} \tilde{O}_{q}\right| 0\right\rangle & =\left\langle 0\left|E_{\Omega}^{(n)}\right| 0\right\rangle \times \delta_{p_{2}, i_{1}} \ldots \delta_{p_{1}, i_{n}} \times \delta_{j_{1} q_{1}} \ldots \delta_{j_{n} q_{n}} \\
& +\left\langle 0\left|\left\{\left(O_{p_{1}}^{(n)}\right)^{\dagger} \ldots\left(O_{p_{2}}^{(1)}\right)^{\dagger}\right\}\left[E_{\Omega_{c}}^{(n)}, O_{i}\right]\right| 0\right\rangle^{*} \times \delta_{j_{1} q_{1}} \ldots \delta_{j_{n} q_{n}} \\
& +\left\langle 0\left|\left[\tilde{O}_{j}^{\dagger}, O_{i}^{\dagger} E_{\Omega}^{(n)} O_{p}\right] \tilde{O}_{q}\right| 0\right\rangle .
\end{aligned}
$$

In right hand side of (B.3), apart from the first piece, all other pieces contain commutators between fields that are mostly supported in disjoint regions of space and hence are small. Thus the first term is the leading piece.

\section{Fermionic fields}

Now we have to keep track of some signs and have to arrange stuff in terms of anticommutators. We treat the cases of even and odd $n$ separately.

Even n. A generic term is still given by (B.3), so all conclusions remain the same, in particular (2.15). Only change is that end of the day we have to break up various commutators in terms of anti-commutators. This can easily be done using the

$$
\left[A, B_{1} \ldots B_{n}\right]=\sum_{p=1}^{n}(-1)^{p-1} B_{1} \ldots B_{p-1}\left\{A, B_{p}\right\} B_{p+1} \ldots B_{n} .
$$

Odd n. In this case we use anti-commutators everywhere, because then we can use the folowing identity to break everything up into anti-commutators

$$
\left\{A, B_{1} \ldots B_{n}\right\}=\sum_{p=1}^{n}(-1)^{p-1} B_{1} \ldots B_{p-1}\left\{A, B_{p}\right\} B_{p+1} \ldots B_{n} .
$$

We break up a generic term as

$$
\left\langle 0\left|\tilde{O}_{j}^{\dagger} O_{i}^{\dagger} E_{\Omega}^{(n)} O_{p} \tilde{O}_{q}\right| 0\right\rangle=-\left\langle 0\left|O_{i}^{\dagger} E_{\Omega}^{(n)} O_{p} \tilde{O}_{j}^{\dagger} \tilde{O}_{q}\right| 0\right\rangle+\left\langle 0\left|\left\{\tilde{O}_{j}^{\dagger}, O_{i}^{\dagger} E_{\Omega}^{(n)} O_{p}\right\} \tilde{O}_{q}\right| 0\right\rangle .
$$


In the first piece of (C.3) we insert complete basis and end up with $\left\langle 0\left|O_{i}^{\dagger} E_{\Omega}^{(n)} O_{p}\right| 0\right\rangle\left\langle 0\left|\tilde{O}_{j}^{\dagger} \tilde{O}_{q}\right| 0\right\rangle$. In this,

$$
\begin{aligned}
\left\langle 0\left|O_{i}^{\dagger} E_{\Omega}^{(n)} O_{p}\right| 0\right\rangle= & \left\langle 0\left|\left(O_{p_{1}}^{(n)}\right)^{\dagger} \ldots\left(O_{p_{2}}^{(1)}\right)^{\dagger} E_{\Omega_{c}}^{(n)} O_{i_{1}}^{(1)} \ldots O_{i_{n}}^{(n)}\right| 0\right\rangle^{*} \\
= & -\left\langle 0\left|\left(O_{p_{1}}^{(n)}\right)^{\dagger} \ldots\left(O_{p_{2}}^{(1)}\right)^{\dagger} O_{i_{1}}^{(1)} \ldots O_{i_{n}}^{(n)} E_{\Omega_{c}}^{(n)}\right| 0\right\rangle^{*} \\
& +\left\langle 0\left|O_{p_{1}}^{(n)} \ldots O_{p_{2}}^{(1)}\left\{E_{\Omega_{c}}^{(n)}, O_{i_{1}}^{(1)} \ldots O_{i_{n}}^{(n)}\right\}\right| 0\right\rangle \\
= & -\left\langle 0\left|E_{\Omega}^{(n)}\right| 0\right\rangle \delta_{i_{1} p_{2}} \ldots \delta_{i_{n} p_{1}}+\left\langle 0\left|O_{p_{1}}^{(n)} \ldots O_{p_{2}}^{(1)}\left\{E_{\Omega_{c}}^{(n)}, O_{i_{1}}^{(1)} \ldots O_{i_{n}}^{(n)}\right\}\right| 0\right\rangle .
\end{aligned}
$$

Gathering all these, we see

$$
\begin{aligned}
\left\langle 0\left|\tilde{O}_{j}^{\dagger} O_{i}^{\dagger} E_{\Omega}^{(n)} O_{p} \tilde{O}_{q}\right| 0\right\rangle= & \left\langle 0\left|E_{\Omega}^{(n)}\right| 0\right\rangle \delta_{i_{1} p_{2}} \ldots \delta_{i_{n} p_{1}} \times \delta_{j_{1} q_{1}} \ldots \delta_{j_{n} q_{n}} \\
& -\left\langle 0\left|O_{p_{1}}^{(n)} \ldots O_{p_{2}}^{(1)}\left\{E_{\Omega_{c}}^{(n)}, O_{i_{1}}^{(1)} \ldots O_{i_{n}}^{(n)}\right\}\right| 0\right\rangle \delta_{j_{1} q_{1}} \ldots \delta_{j_{n} q_{n}} \\
& +\left\langle 0\left|\left\{\tilde{O}_{j}^{\dagger}, O_{i}^{\dagger} E_{\Omega}^{(n)} O_{p}\right\} \tilde{O}_{q}\right| 0\right\rangle
\end{aligned}
$$

Since the leading piece remains the same, (2.15) goes through. The details of the corrections terms is different from those in B, although the difference is only quantitative.

Open Access. This article is distributed under the terms of the Creative Commons Attribution License (CC-BY 4.0), which permits any use, distribution and reproduction in any medium, provided the original author(s) and source are credited.

\section{References}

[1] A. Einstein, B. Podolsky and N. Rosen, Can quantum mechanical description of physical reality be considered complete?, Phys. Rev. 47 (1935) 777 [InSPIRE].

[2] S. Ryu and T. Takayanagi, Holographic derivation of entanglement entropy from AdS/CFT, Phys. Rev. Lett. 96 (2006) 181602 [hep-th/0603001] [INSPIRE].

[3] S. Ryu and T. Takayanagi, Aspects of Holographic Entanglement Entropy, JHEP 08 (2006) 045 [hep-th/0605073] [INSPIRE].

[4] H. Casini and M. Huerta, A finite entanglement entropy and the c-theorem, Phys. Lett. B 600 (2004) 142 [hep-th/0405111] [INSPIRE].

[5] H. Casini and M. Huerta, A c-theorem for the entanglement entropy, J. Phys. A 40 (2007) 7031 [cond-mat/0610375] [INSPIRE].

[6] G. Vidal, J.I. Latorre, E. Rico and A. Kitaev, Entanglement in quantum critical phenomena, Phys. Rev. Lett. 90 (2003) 227902 [quant-ph/0211074] [INSPIRE].

[7] A. Kitaev and J. Preskill, Topological entanglement entropy, Phys. Rev. Lett. 96 (2006) 110404 [hep-th/0510092] [INSPIRE].

[8] M. Levin and X.-G. Wen, Detecting Topological Order in a Ground State Wave Function, Phys. Rev. Lett. 96 (2006) 110405 [INSPIRE].

[9] P. Caputa, M. Nozaki and T. Takayanagi, Entanglement of local operators in large- $N$ conformal field theories, PTEP 2014 (2014) 093B06 [arXiv: 1405.5946] [INSPIRE]. 
[10] M. Nozaki, T. Numasawa and T. Takayanagi, Quantum Entanglement of Local Operators in Conformal Field Theories, Phys. Rev. Lett. 112 (2014) 111602 [arXiv:1401.0539] [INSPIRE].

[11] N. Shiba, Entanglement Entropy of Disjoint Regions in Excited States: An Operator Method, JHEP 12 (2014) 152 [arXiv:1408.0637] [INSPIRE].

[12] S.W. Hawking, Particle Creation by Black Holes, Commun. Math. Phys. 43 (1975) 199 [Erratum ibid. 46 (1976) 206] [INSPIRE].

[13] S.W. Hawking, Breakdown of Predictability in Gravitational Collapse, Phys. Rev. D 14 (1976) 2460 [inSPIRE].

[14] S.D. Mathur, The information paradox: A pedagogical introduction, Class. Quant. Grav. 26 (2009) 224001 [arXiv:0909.1038] [INSPIRE].

[15] A. Almheiri, D. Marolf, J. Polchinski and J. Sully, Black Holes: Complementarity or Firewalls?, JHEP 02 (2013) 062 [arXiv:1207.3123] [INSPIRE].

[16] P. Zanardi, Quantum entanglement in fermionic lattices, Phys. Rev. A 65 (2002) 042101 [quant-ph/0104114].

[17] P. Zanardi and X. Wang, Fermionic entanglement in itinerant systems J. Phys. A 35 (2002) 7947 [quant-ph/0201028].

[18] K. Eckert, J. Schliemann, D. Bruß and M. Lewenstein, Quantum Correlations in Systems of Indistinguishable Particles, Annals Phys. 299 (2002) 88 [quant-ph/0203060].

[19] Y. Shi, Quantum entanglement of identical particles, Phys. Rev. A 67 (2003) 024301 [INSPIRE].

[20] J.K. Korbicz and M. Lewenstein, Group-theoretical approach to entanglement, Phys. Rev. A 74 (2006) 022318 [quant-ph/0601189].

[21] A.P. Balachandran, T.R. Govindarajan, A.R. de Queiroz and A.F. Reyes-Lega, Entanglement and Particle Identity: A Unifying Approach, Phys. Rev. Lett. 110 (2013) 080503 [arXiv:1303.0688] [inSPIRE].

[22] G. Ghirardi, L. Marinatto and T. Weber, Entanglement and Properties of Composite Quantum Systems: a Conceptual and Mathematical Analysis, J. Stat. Phys. 108 (2002) 49 [quant-ph/0109017].

[23] R. Horodecki, P. Horodecki, M. Horodecki and K. Horodecki, Quantum entanglement, Rev. Mod. Phys. 81 (2009) 865 [quant-ph/0702225] [INSPIRE].

[24] C.H. Bennett, H.J. Bernstein, S. Popescu and B. Schumacher, Concentrating partial entanglement by local operations, Phys. Rev. A 53 (1996) 2046 [quant-ph/9511030] [INSPIRE].

[25] H. Reeh and S. Schlieder, Bemerkungen zur Unitäräquivalenz von Lorentzinvarianten Feldern Nuovo Cim. 22 (1961) 1051.

[26] H. Li and F. Haldane, Entanglement Spectrum as a Generalization of Entanglement Entropy: Identification of Topological Order in Non-Abelian Fractional Quantum Hall Effect States, Phys. Rev. Lett. 101 (2008) 010504 [INSPIRE].

[27] J.R. Grittings and A.J. Fisher, Describing mixed spin-space entanglement of pure states of indistinguishable particles using an occupation-number basis, Phys. Rev. A 66 (2002) 032305 [quant-ph/0202051]. 
[28] H.M. Wiseman and J.A. Vaccaro, Entanglement of Indistinguishable Particles Shared between Two Parties, Phys. Rev. Lett. 91 (2003) 097902 [quant-ph/0210002].

[29] M.R. Dowling, A.C. Doherty and H.M. Wiseman, Entanglement of indistinguishable particles in condensed-matter physics, Phys. Rev. A 73 (2006) 052323 [quant-ph/0601079].

[30] H.W. Lee and J. Kim, Quantum teleportation and Bell's inequality using single-particle entanglement, Phys. Rev. A 63 (2000) 012305 [quant-ph/0007106]. 